\title{
Publisher Correction: Measuring the ionisation fraction in a jet from a massive protostar
}

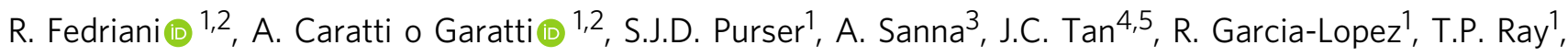 \\ D. Coffey (iD ${ }^{1,2}$, B. Stecklum ${ }^{6} \&$ M. Hoare ${ }^{7}$
}

Correction to: Nature Communications https://doi.org/10.1038/s41467-019-11595-x, published online 9 August 2019.

The original version of this Article contained an error in last sentence of the legend to Table 4, which incorrectly read 'a Assuming a tangential velocity of $150-300 \mathrm{~km}^{-1}$ from the proper motions of ref. ${ }^{22}$ ' The correct version states ' $150-300 \mathrm{~km} \mathrm{~s}$ ' ' $150-300 \mathrm{~km}^{-1}$ '.

The original version also contained errors in Table 5, in which the headings of the fifth, sixth and seventh rows incorrectly read, ' $\dot{M}$, $\left(\times 10^{-6} M_{\odot} \mathrm{y}^{-1}\right)^{\prime}$, ' $\dot{M}_{\text {ejec }}\left(\times 10^{-5} M_{\odot} \mathrm{y}^{-1}\right)^{\prime}$ ' and ' $\dot{P}_{\text {ionised }}\left(\times 10^{-4} M_{\odot} \mathrm{y}^{-1} \mathrm{~km}^{-1}\right)$ ', instead of the correct ' $\dot{M}_{\text {ionised }}\left(\times 10^{-6} M_{\odot} \mathrm{yr}-1\right)^{-}$, ' $\dot{M}_{\text {ejec }}$ $\left(\times 10^{-5} M_{\odot} \mathrm{yr}^{-1}\right)$ ' and ' $\dot{P}_{\text {ionised }}\left(\times 10^{-4} M_{\odot} \mathrm{yr}^{-1} \mathrm{~km} \mathrm{~s}^{-1}\right)$ ', respectively.

The last sentence of the legend to Table 5 originally incorrectly read 'a Assuming a tangential velocity of $150-300 \mathrm{~km}^{-1}$ from the proper motions of ref. ${ }^{22}$ ' The correct version states ' $150-300 \mathrm{~km} \mathrm{~s}^{-1}$ ' in place of ' $150-300 \mathrm{~km}^{-1}$ '.

The original version also contained an error in the third sentence of the first paragraph of the 'Ionised mass-loss rate on source' section of the Methods, which incorrectly read 'From our observations we obtain the following parameters for core B: $S_{5.8}=0.794 \pm 0.03 \mathrm{mJy}$, $v=5.8 \mathrm{GHz}, v_{j}=600 \pm 100 \mathrm{~km}^{-1}, \theta_{0}=52.3^{\circ} \pm 4.4^{\circ}, D=2.2 \mathrm{kpc}, T=10,000 \mathrm{~K}$, the resulting ionised mass-loss rate is $1.81 \pm 0.33 \times 10^{-6}$ $\mathrm{M}_{\odot} \mathrm{yr}^{-1}$, consistent with ref. ${ }^{22}$.' The correct version states ' $v_{j}=600 \pm 100 \mathrm{~km} \mathrm{~s}^{-1}$ ' in place of ' $v_{j}=600 \pm 100 \mathrm{~km}^{-1}$ '.

This has been corrected in both the PDF and HTML versions of the Article.

Published online: 03 September 2019

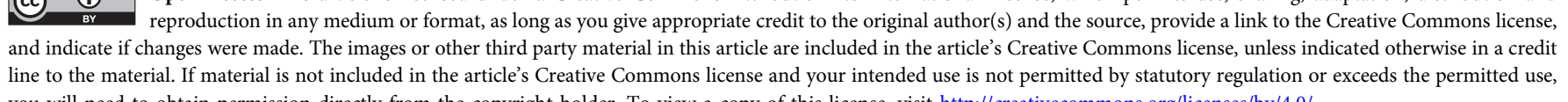
you will need to obtain permission directly from the copyright holder. To view a copy of this license, visit http://creativecommons.org/licenses/by/4.0/.

(C) The Author(s) 2019

\footnotetext{
${ }^{1}$ Dublin Institute for Advanced Studies, School of Cosmic Physics, Astronomy \& Astrophysics Section, 31 Fitzwilliam Place, Dublin D02 XF86, Ireland.

${ }^{2}$ School of Physics, University College Dublin, Belfield, Dublin 4, Ireland. ${ }^{3}$ Max-Planck-Institut für Radioastronomie, Auf dem Hügel 69, 53121 Bonn, Germany. ${ }^{4}$ Department of Space, Earth \& Environment, Chalmers University of Technology, SE-412 93 Gothenburg, Sweden. ${ }^{5}$ Department of Astronomy, University of Virginia, 530 McCormick Road, Charlottesville, VA 22904-4325, USA. ${ }^{6}$ Thüringer Landessternwarte Tautenburg, Sternwarte 5, 07778 Tautenburg, Germany. ${ }^{7}$ School of Physics and Astronomy, University of Leeds, Leeds LS2 9JT, UK. Correspondence and requests for materials should be addressed to R.F. (email: fedriani@cp.dias.ie)
} 